\title{
Quasi-cosmological traversable wormholes in $f(R)$ gravity
}

\author{
Hanif Golchin $^{\mathrm{a}}$, Mohammad Reza Mehdizadeh ${ }^{\mathrm{b}}$ \\ Faculty of Physics, Shahid Bahonar University of Kerman, PO Box 76175, Kerman, Iran
}

Received: 6 March 2019 / Accepted: 9 September 2019 / Published online: 20 September 2019

(C) The Author(s) 2019

\begin{abstract}
In this paper, we study traversable wormholes in the context of $f(R)$ gravity. Exact solutions of traversable wormholes are found by imposing the nonconstant Ricci scalar. These solutions asymptotically match spherical, flat and hyperbolic FRW metric. By choosing some static $f(R)$ gravity models, we verify the standard energy conditions for the asymptotically spherical, flat and hyperbolic wormhole solutions. Unlike the Einstein gravity, we find that in the context of $f(R)$ modified gravity, the asymptotically spherical, flat and hyperbolic wormhole solutions can respect the null energy condition (NEC) at the wormhole throat and near that. We find that in some static $f(R)$ models, asymptotically flat and hyperbolic wormholes respect the weak energy condition (WEC) through the whole space.
\end{abstract}

\section{Introduction}

Traversable wormholes are throatlike geometrical structures which connect two separate and distinct regions of spacetimes and have no horizon or singularity. There are some hints in the paper of Flamm [1], to such spatial geometry. In 1935, Einstein and Rosen [2] firstly obtained the wormhole solutions as bridge model of a particle. The study of Lorentzian wormholes in the context of general relativity (GR) was stimulated by the significant paper of Morris and Thorne in 1988 [3,4]. A fundamental ingredient in wormhole physics is the flaring-out condition of the throat, which in GR entails the violation of the NEC. Matter that violates the NEC is denoted exotic matter.

A very important challenge in wormhole scenarios is the establishment of standard energy conditions. In this regard, various methods have been proposed in the literature that deal with the issue of energy conditions within wormhole settings. Work along this line has been done in dynamical wormholes

\footnotetext{
a e-mail: h.golchin@uk.ac.ir

be-mail: mehdizadeh.mr@uk.ac.ir
}

[5-7] and thin-shell wormholes [8], where the supporting matter is concentrated on the wormholes throat. In the context of modified theories of gravity, the presence of higherorder terms in curvature would allow for building thin-shell wormholes supported by ordinary matter [9-11]. Recently, many people try to build and study wormhole solutions within the framework of modified gravity for instance, wormhole solutions in BransDicke theory [12-15], Born-Infeld theory $[16,17]$, Einstein-Gauss-Bonnet theory [18,19], KaluzaKlein gravity [20,21] and scalar-tensor gravity [22] and Einstein-Cartan theory [23-25].

In recent years, theories of modified gravity have been studied involving cosmological objects like black holes, gravastars, strange stars and wormholes. Such theories are able to explain accelerating expansion of the universe and also solve the dark matter problem [26-28]. A well-known theory of modified gravity is $\mathrm{f}(\mathrm{R})$ gravity which modifies GR by replacing the gravitational action $\mathrm{R}$ by an arbitary function $f(R)$, where $R$ is the Ricci scalar. These models can be demonstrated to cause acceleration [29-35]. It was found that higher-order curvature invariants can satisfy the energy conditions in $f(R)$ gravity [36-38]. The power-law $R^{m}$ gravity is also studied in $[39,40]$, the new static wormholes in $\mathrm{f}(\mathrm{R})$ theory using the non-commutative geometry are built in $[41,42]$ and the cosmological evolution of wormhole solutions is investigated in $[43,44]$. Recently, the junction conditions in $f(R)$ applied to the construction of thin-shell wormholes [45-47] and pure double layer bubbles [48]. The static wormhole geometries have also been theorized within curvature-matter coupling theories such as $f(R, T)[49,50]$.

Lorentzian wormhole solutions were also investigated in viable $f(R)$ modified theories of gravity [51], which are consistent with observations of the solar system and cosmological evolution in [52-57]. These wormholes were found in specific $f(R)$ models which are considered to reproduce realistic scenarios of cosmological evolution based on the accelerated expansion of the universe. it was shown that the WEC holds at the vicinity of the throat for certain ranges of the free 
parameters of the theory. These asymptotically flat solutions analysed with the simple choices of shape function which WEC is satisfied at a specific point in space.

A large class of static black hole solutions with non constant Ricci scalar and traversable wormholes with constant Ricci scalar have also investigated in the background of $f(R)$ gravity [58,59]. The existence of wormhole solutions in scalar-tensor and $f(R)$ gravity was studied in $[60,61]$ and it was shown that static wormhole solutions in these theories can be obtained with matter respecting the WEC when the effective gravitational constant is negative (which means the anti-gravitational property of the solutions) in some regions of space.

In this work, our aim is to obtain the wormhole solutions with non constant Ricci scalar in the context of viable $f(R)$ gravity. This paper is organized as follows: after this introduction, we will present the basic field equations of $f(R)$ gravity . In Sec. III, we will derive the properties of wormholes solutions within the framework of $f(R)$ gravity and discuss about the energy conditions. The final section is devoted to our concluding remarks.

\section{$2 \mathbf{f}(\mathrm{R})$ gravity wormholes}

The action of $f(R)$ modified theories of gravity is given by

$S=\frac{1}{2 \kappa} \int d^{4} x \sqrt{-g} f(R)+\int d^{4} x \sqrt{-g} \mathcal{L}_{m}\left(g_{\mu \nu}, \psi\right)$,

where $\kappa=8 \pi G$ and $\mathcal{L}_{m}$ is the matter Lagrangian density, in which the matter field $\psi$ is minimally coupled to the metric $g_{\mu \nu}$. Throughout this work for notational simplicity we consider $\kappa=1$. One can find the field equation by varying the action (1) with respect to metric $g^{\mu \nu}$ as

$F R_{\mu \nu}-\frac{1}{2} f g_{\mu \nu}-\nabla_{\mu} \nabla_{\nu} F+g_{\mu \nu} \square F=T_{\mu \nu}^{m}$,

and the trace of the field equation

$F R-2 f+3 \square F=T$,

where $F=d f / d R$. Substituting the trace equation in (2) and re-organizing the terms, one can write the field equation as [62]

$G_{\mu \nu} \equiv R_{\mu \nu}-\frac{1}{2} R g_{\mu \nu}=T_{\mu \nu}^{c}+\tilde{T}_{\mu \nu}^{m}$.

In the above, the two terms in the right hand side of the field equation are in the form

$T_{\mu \nu}^{c}=\frac{1}{F}\left[\nabla_{\mu} \nabla_{\nu} F-\frac{1}{4} g_{\mu \nu}(R F+\square F+T)\right]$,

$\tilde{T}_{\mu \nu}^{m}=T_{\mu \nu}^{m} / F$.

In this background, we are going to study the static and spherically symmetric traversable wormholes which is given by the following metric ${ }^{1}$

$d s^{2}=-d t^{2}+\frac{d r^{2}}{1-b(r) / r}+r^{2}\left(d \theta^{2}+\sin ^{2} \theta d \phi^{2}\right)$.

The Ricci scalar for the above wormhole geometry is

$R=\frac{2 b^{\prime}(r)}{r^{2}}$

where " " denotes derivative with respect to the radial coordinate $r$. As in [62] we choose the energy-momentum tensor of anisotropic distribution of matter

$T_{\mu \nu}=\left(\rho+p_{t}\right) U_{\mu} U_{\nu}+p_{t} g_{\mu \nu}+\left(p_{r}-p_{t}\right) \chi_{\mu} \chi_{\nu}$,

where $U^{\mu}$ is the four-velocity and $\chi^{\mu}=\sqrt{1-b(r) / r} \delta_{r}^{\mu}$ is the unit spacelike vector in the radial direction. $\rho(r)$ is the energy density and $p_{r}(r), p_{t}(r)$ are radial and transverse pressure respectively, so the energy-momentum tensor takes to the form $T_{\nu}^{\mu}=\operatorname{diag}\left[-\rho, p_{r}, p_{t}, p_{t}\right]$. Thus the field equation (4) leads to the following relationships

$$
\begin{aligned}
\frac{b^{\prime}}{r^{2}} & =\frac{\rho}{F}+\frac{H}{F} \\
-\frac{b}{r^{3}} & =\frac{p_{r}}{F}+\frac{1}{F}\left\{\left(1-\frac{b}{r}\right)\left[F^{\prime \prime}-F^{\prime} \frac{b^{\prime} r-b}{2 r^{2}(1-b / r)}\right]-H\right\} \\
\frac{b-b^{\prime} r}{2 r^{3}} & =\frac{p_{t}}{F}+\frac{1}{F}\left[\left(1-\frac{b}{r}\right) \frac{F^{\prime}}{r}-H\right]
\end{aligned}
$$

where $H(r)=\frac{1}{4}(F R+\square F+T)$. By solving the above system, one finds the following expressions [62]

$$
\begin{aligned}
\rho & =\frac{F b^{\prime}}{r^{2}}, \\
p_{r} & =-\frac{b F}{r^{3}}+\frac{F^{\prime}}{2 r^{2}}\left(b^{\prime} r-b\right)-F^{\prime \prime}\left(1-\frac{b}{r}\right), \\
p_{t} & =-\frac{F^{\prime}}{r}\left(1-\frac{b}{r}\right)+\frac{F}{2 r^{3}}\left(b-b^{\prime} r\right) .
\end{aligned}
$$

We are interested in studying inhomogeneous spacetimes which merge smoothly to the cosmological background. Therefore, we may consider the Ricci scalar of the wormhole geometry as

$R=6 c_{1}+\frac{6 c_{2}}{r^{n}}$,

where $c_{1}, c_{2}$ and $n$ are free parameters. In the following section we consider some choices for these free parameters and study the corresponding wormhole solutions in some different $f(R)$ backgrounds.

1 The static and spherically symmetric wormhole geometry is given by $d s^{2}=-e^{2 \Phi(r)} d t^{2}+\frac{d r^{2}}{1-b(r) / r}+r^{2}\left(d \theta^{2}+\sin ^{2} \theta d \phi^{2}\right)$.

The solution is traversable when $\Phi(r)$ is finite. In the following we set $\Phi(r)=0$. 


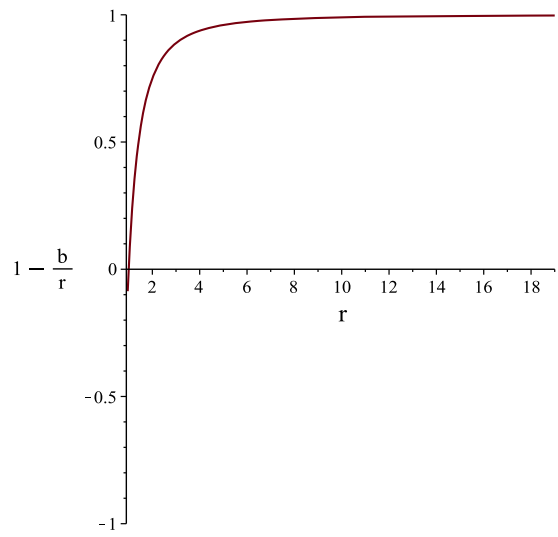

(a)

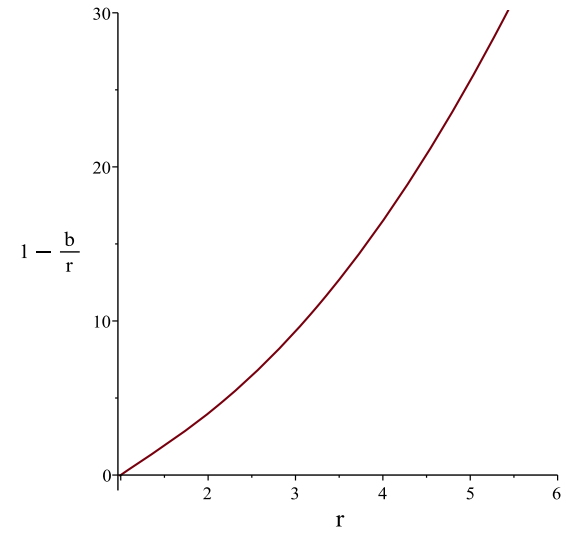

(b)

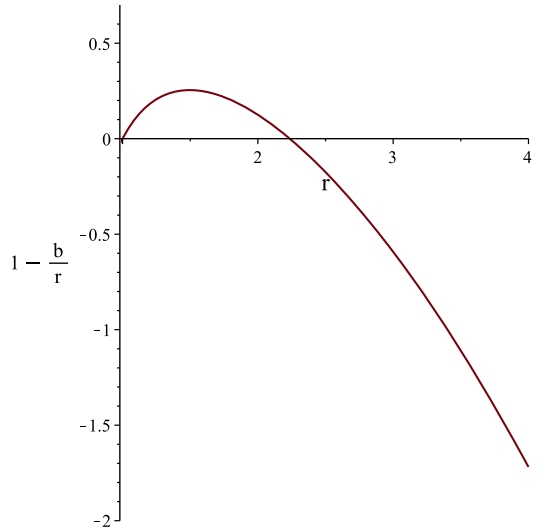

(c)

Fig. 1 a-c Show the asymptotically flat $\left(c_{1}=0\right)$, asymptotically hyperbolic $\left(c_{1}=-1\right)$ and asymptotically spherical $\left(c_{1}=1\right)$ wormhole solutions respectively. Wormhole throat is chosen at $r_{0}=1$, we also set $n=4$ in these figures

\section{Traversable wormhole solutions in $f(R)$ models}

We start this section by finding wormhole solutions with Ricci scalar in the form (13). Combining (7) and (13) one can find the shape function as

$b(r)=r^{3}\left(c_{1}+\frac{3 c_{2}}{3-n} r^{-n}\right)+c_{3}$,

where $c_{3}$ is the integration constant. This wormhole solution by choosing $c_{2}=0$ is obtained in [59]. In the following we set the integration constant to zero. The shape function $b(r)$ for a wormhole solution, should satisfy the following conditions:

i) $b\left(r_{0}\right)=r_{0}$,

ii) $b^{\prime}\left(r_{0}\right)<1$,

iii) $1-\frac{b(r)}{r}>0$.

Inserting condition $i$ ), one can find $c_{2}$ in terms of $r_{0}, c_{1}$ and $n$; so it is possible to rewrite the shape function as

$b(r)=\left[\left(-r_{0}^{n} c_{1}+r_{0}^{n-2}\right) r^{3-n}+c_{1} r^{3}\right]$.

We consider the wormhole solutions with three values $c_{1}=0, \pm 1$. The shape function for these values are depicted in Fig. 1. It is easy to check that in the case of $c_{1}=0,-1$ the condition ii) in (15) is satisfied for $n>2$ and in the case of $c_{1}=1$ condition $\left.i i\right)$ is satisfied when $n>2.4$.

Setting the shape function into the form (16), one can write

$\frac{b(r)}{r}=c_{1} r^{2}+\left(r_{0}^{n-2}-c_{1} r_{0}^{n}\right) r^{2-n}$,

note that due to the condition ii) we should insert $n>2$ in the above, so the second term in (17) falls down at large $r$. Remembering the wormhole metric (6), one deduces that the wormhole solutions with $c_{1}=-1, c_{1}=0$ and $c_{1}=1$ at large $r$ match the hyperbolic, flat and spherical FRW universe respectively, so we call them asymptotically flat $\left(c_{1}=0\right)$, asymptotically hyperbolic $\left(c_{1}=-1\right)$ and asymptotically spherical $\left(c_{1}=1\right)$ wormhole solutions.

In the framework of Einstein gravity, the traversable wormhole geometry (6), violates the weak energy condition WEC $1: \rho>0$, WEC2: $\rho+p_{t}>0$, WEC $3: \rho+p_{r}>0$ and the null energy condition WEC2: $\rho+p_{t}>0$, WEC3: $\rho+p_{r}>0$. Substituting $\rho, p_{r}$ and $p_{t}$ given in (10)-(12) and the shape function (16), one can rewrite the requirements of WEC as

WEC1: $\left[3 c_{1}+(n-3) r^{-n}\left(c_{1} r_{0}^{n}-r_{0}^{n-2}\right)\right] F>0$,

WEC $2: \frac{1}{2 r}\left[F\left(24 c_{1} r+(4-n)\left(r_{0}^{n-2}-6 c_{1} r_{0}^{n}\right) r^{1-n}\right)\right.$

$$
\left.+2 F^{\prime}\left(c_{1} r^{2}-1+\left(r_{0}^{n-2}-6 c_{1} r_{0}^{n}\right) r^{2-n}\right)\right]>0,
$$

WEC $3: F\left[12 c_{1}-(n-2)\left(r_{0}^{n-2}-6 c_{1} r_{0}^{n}\right) r^{-n}\right]$

$$
\begin{aligned}
& +\frac{1}{2} F^{\prime}\left[12 c_{1} r+\left(6 c_{1} r_{0}^{n}-r_{0}^{n-2}\right)(n-2) r^{1-n}\right] \\
& +F^{\prime \prime}\left[6 c_{1} r^{2}-1+\left(r_{0}^{n-2}-6 r_{0}^{n} c_{1}\right)\right] r^{2-n}>0,
\end{aligned}
$$

where $F=d f(R) / d R$ and " ' " denotes derivative with respect to the radial coordinate $r$.

The conditions of stability and absence of ghosts are discussed in $[60,61]$. It is shown that in a scalar-tensor theory of gravity formulated in the Jordan frame with Lagrangian

$L=\frac{1}{2}\left[f(\phi) R+h(\phi) g^{\mu v} \phi_{, \mu} \phi_{, v}-2 U(\phi)\right]+L_{m}$,

where $f, h$ and $U$ are arbitrary functions and $L_{m}$ is the matter Lagrangian, there is no static wormhole solution that satisfy the null energy conditions when $f(\phi)>0$ and $f(\phi) h(\phi)+$ $\frac{3}{2}\left(\frac{d f}{d \phi}\right)^{2}>0$. In the case of $f(R)$ theories of modified gravity $h(\phi)=0$ and $f(\phi)=F=d f(R) / d R$ [61], so the above nonexistence theorem holds for 


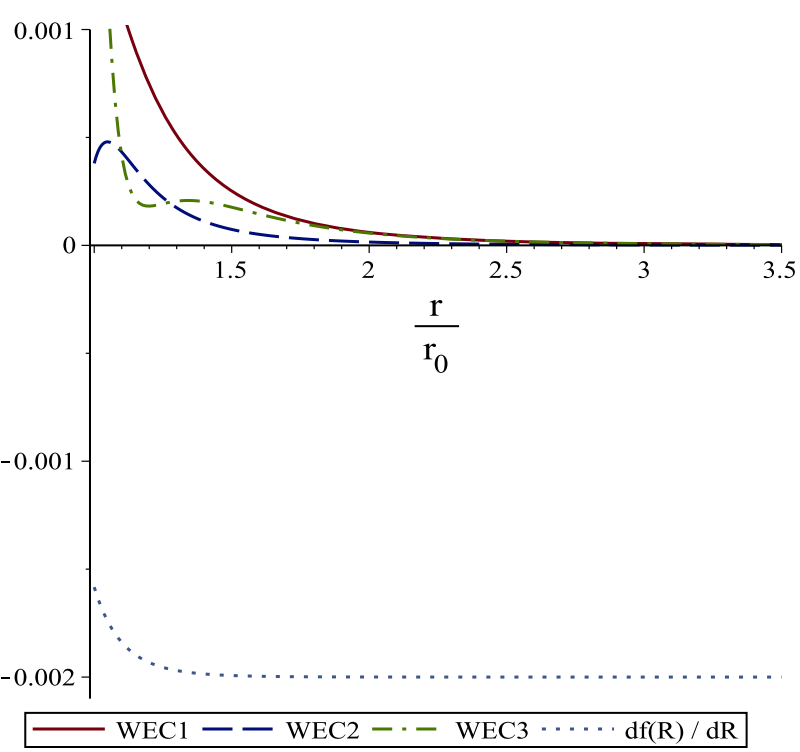

(a)

Fig. $2 F=d f(R) / d R$ and weak energy condition requiements for a wormhole solution of the Tsujikawa model with $n=5$ are depicted in a. It is obvious that $F<0$, so the condition of wormhole nonexistence is violated. It is also clear that WEC is respected at the throat $r_{0}$ and

$F=\frac{d f(R)}{d R}>0, \quad \frac{d^{2} f(R)}{d R^{2}} \neq 0$.

In the following we consider the asymptotically flat $\left(c_{1}=\right.$ $0)$, asymptotically hyperbolic $\left(c_{1}=-1\right)$ and asymptotically spherical $\left(c_{1}=1\right)$ wormhole solutions in the background of $f(R)$ modified gravity. We are interested to the wormholes that respect the requirements of WEC, in the other word, we look for the solutions which satisfy (18), the conditions $i i)$, iii) in (15) and violate the nonexistence theorem (20) simultaneously ${ }^{2}$. Note that the regions where $d f(R) / d R<0$ are anti-gravitational, in the sense that the effective gravitational constant is negative $[60,61]$.

\subsection{The case of $c_{1}=0$}

For a wormhole solution, as we mentioned above, the second term in (17) falls down at large $r$, therefore in the case of $c_{1}=0$ the traversable wormhole solution (6) matches the flat FRW metric at large $r$. At this stage we consider such asymptotically flat wormholes in the background of some static $f(R)$ gravity models. As we will see, the $f(R)$ modified gravity wormhole solutions in many cases satisfy the null or weak energy condition.

\footnotetext{
$\overline{2}$ Note that by $d^{2} f(R) / d R^{2}=0$ in (20), the nonexistence theorem violates just in one or some points instead, the $F<0$ violates this theorem in a region at space. Hence in the following we search for $F<0$ to check the violation of this nonexistence theorem.
}

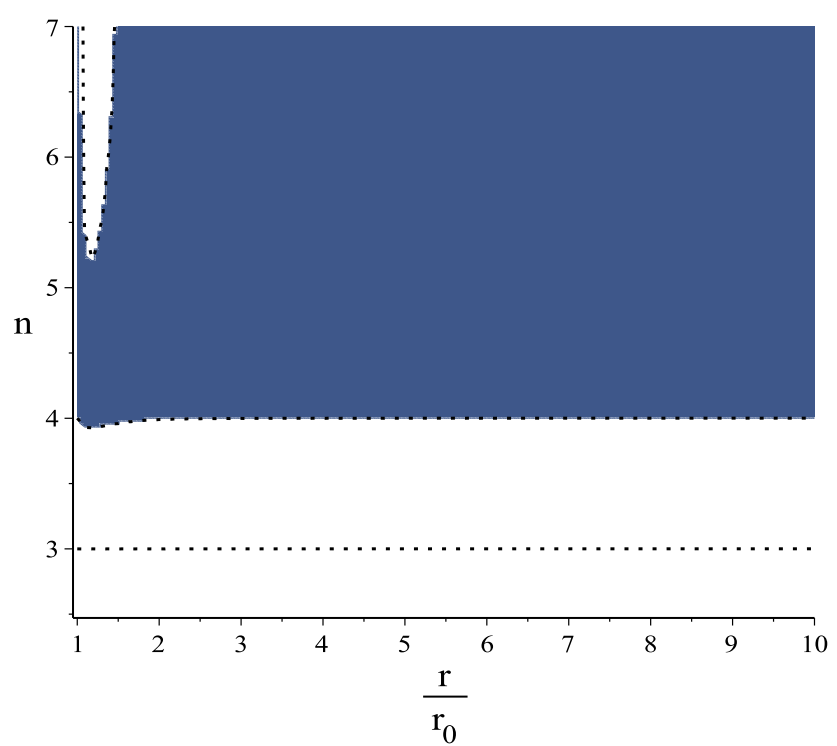

(b)

beyond it. In the blue region of $\mathbf{b}, F<0$ and (18) are simultaneously satisfied, so this region shows traversable asymptotically flat wormholes of the Tsujikawa model, that respect the WEC. In these figures we set $\mu=1.02, R_{*}=0.1$ and $r_{0}=25$

1) The Tsujikawa model [63-65]

One of $f(R)$ models which is consistent with cosmological and local gravity conditions, proposed by Tsujikawa. In this viable model, the $f(R)$ considered as

$f(R)=R-\mu R_{*} \tanh \left(\frac{R}{R_{*}}\right)$,

where $0.905<\mu<1$ and $R_{*}$ is a free small positive parameter [63]. For this model one can easily finds $F=1-\mu+\mu \tanh ^{2}\left(R / R_{*}\right)$. It is obvious that $F$ takes positive values when $\mu<1$, this means that by choosing $\mu$ in the consistent range $0.905<\mu<1$, due to (20), there is no static wormhole solution in this model that satisfy the NEC (and so WEC). However by taking $\mu$ at the neighborhood of this range, one can find wormholes that respect WEC. In Fig. 2a we have depicted $F$ in (20) and the requirements of WEC (18) for a solution with $\mu=1.02$ and $n=5$. It is obvious in this figure that $F<0$, therefore the condition of nonexistence theorem (20) is violated. The figure also shows that WEC is respected at the wormhole throat $r_{0}$.

It is worth to mention that in this model, there is a 4dimensional parameter space for the wormhole solutions which is made of $\mu, R_{*}, r_{0}$ and $n$. Among these parameters, $\mu, R_{*}$ are limited by the model [63], we also measure the radial distance $r$, in the unit of wormhole throat $r_{0}$ (the WEC1,2,3 in Fig. 2a are depicted in term of $r / r_{0}$ ). Now we are going to find the parameter $n$ in a manner that wormhole solutions respect WEC through the space. In the other 


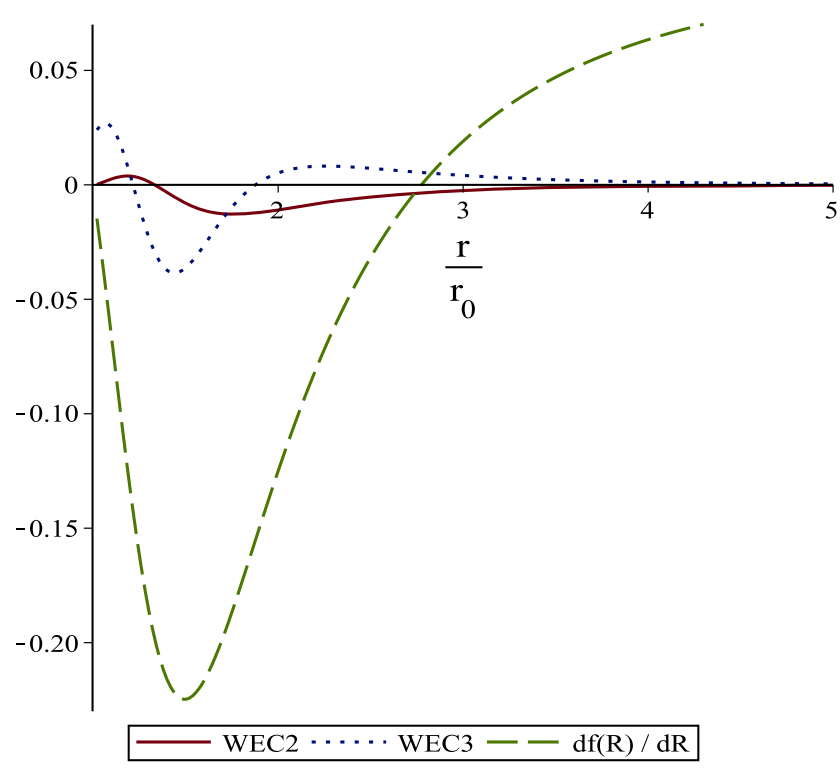

(a)

Fig. 3 Setting $n=2.8$ in a, we can see that $d f(R) / d R<0$ around the throat and the obtained wormhole solution of $\mathrm{Hu}-\mathrm{Sawicki}$ model respect $\mathrm{NEC}$ at $r_{0}$. The blue region in $\mathbf{b}$ corresponds to traversable asymptoti-

word we search for regions in $n-r / r_{0}$ diagram that (18) and conditions $i i), i i i)$ in (15) are satisfied and $F$ in (20) takes a negative value simultaneously.

Figure $2 \mathrm{~b}$ shows $n-r / r_{0}$ diagram. The blue region in this figure corresponds to asymptotically flat traversable wormhole solutions in the background of (21), which respect the WEC. It is obvious that by choosing $4<n<5$.2, the WEC

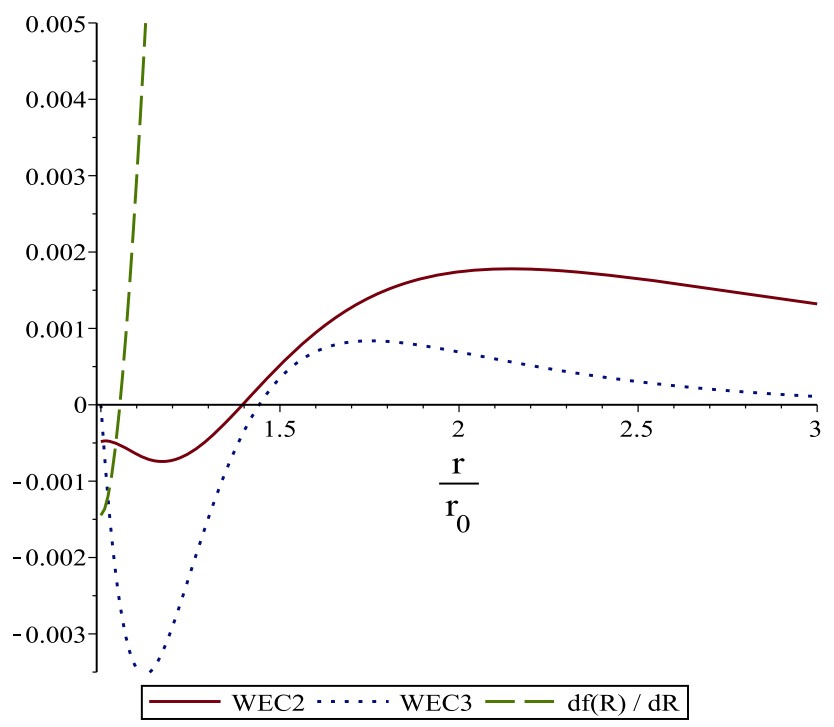

Fig. 4 It is clear that $d f(R) / d R<0$ at the throat, but the wormhole solutions in Starobinsky model do not respect NEC at the throat $r_{0}$. In this figures we set $q=2, \lambda=0.98, R_{*}=0.01$ and $r_{0}=15$

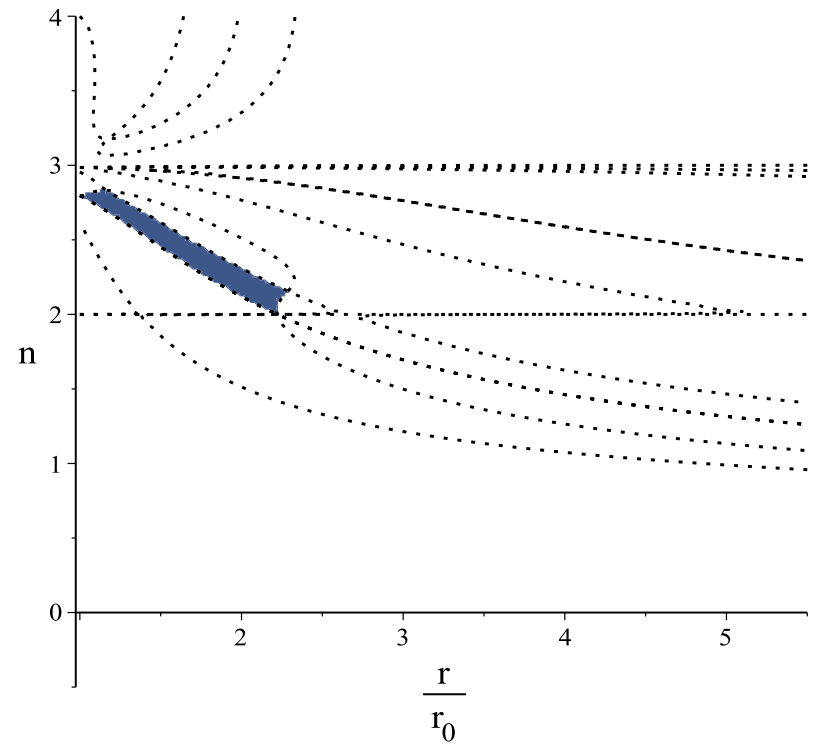

(b)

cally flat wormholes of the Hu-Sawicki model, which respect NEC. In these figures we set $m=1, \mu=5, R_{*}=0.001$ and $r_{0}=15$

is respected at the throat $r_{0}$ and through the whole space outside that. Note that unlike the Einstein gravity, considering $f(R)$ gravity model in the form (21), it is possible to find traversable wormhole solutions that respect the WEC at the throat and beyond that.

2) Hu-Sawicki model

The second $f(R)$ model which satisfies both cosmological and local gravity constraints proposed by $\mathrm{Hu}$ and Sawicki $[65,66]$. This model is in the form

$f(R)=R-\mu R_{*} \frac{\left(R / R_{*}\right)^{2 m}}{\left(R / R_{*}\right)^{2 m}+1}$,

where $m, R_{*}$ and $\mu$ are positive parameters. It is shown in [63] that $R_{*}$ takes a small positive value and for $m=1$ one should insert $\mu \geq 8 \sqrt{3} / 9$. Similar to the previous case, we are looking for the wormhole solutions of this $f(R)$ model which respect the NEC or WEC. In Fig. 3a by setting $\mu=5$ and $n=2.8$, we have depicted $F$ in (20) and WEC2,3 (18). It is clear from the figure that around the throat $r_{0}, F<0$ and WEC2,3 are positive, which means that this wormhole solution respect the null energy conditions.

Figure $3 \mathrm{~b}$ shows the $n-r / r_{0}$ diagram for this model. The blue zone in this figure is the region that $F<0, \operatorname{WEC} 2>0$, WEC3 $>0$ and ii), iii) in (15) are satisfied simultaneously. This means that traversable asymptotically flat wormhole solutions in this model respect the NEC around the throat $r_{0}$ when $2<n<3$ and by setting $n \sim 2.8$ the NEC is respected at the throat. 


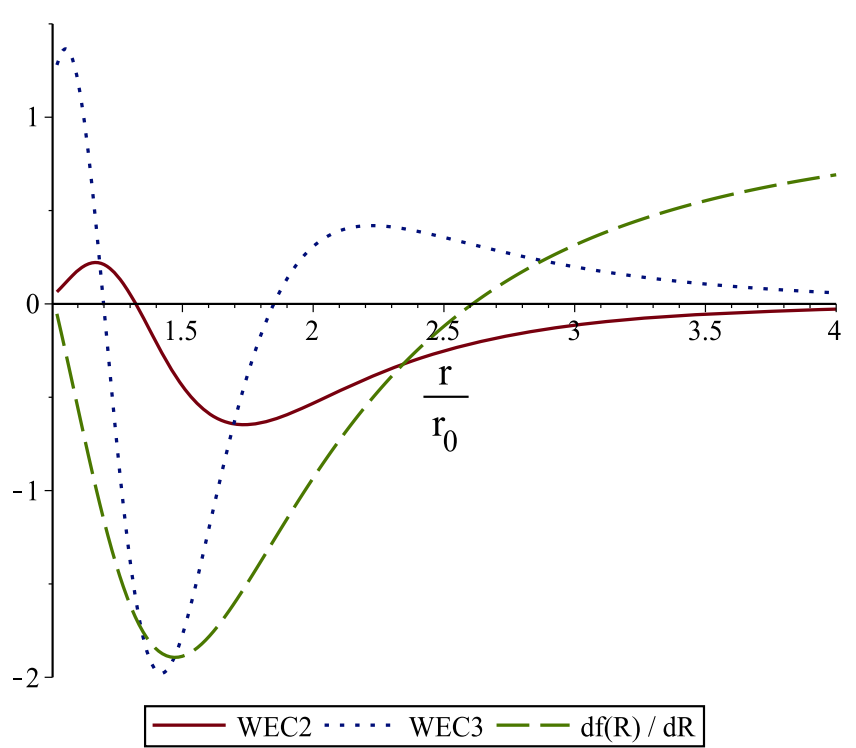

(a)

Fig. 5 a Shows that $d f(R) / d R<0$ and the NEC is satisfied at the throat for the wormhole solution of the Nojiri-Odintsov model with $n=2.8$. According to $\mathbf{b}$ asymptoticly flat traversable wormholes in

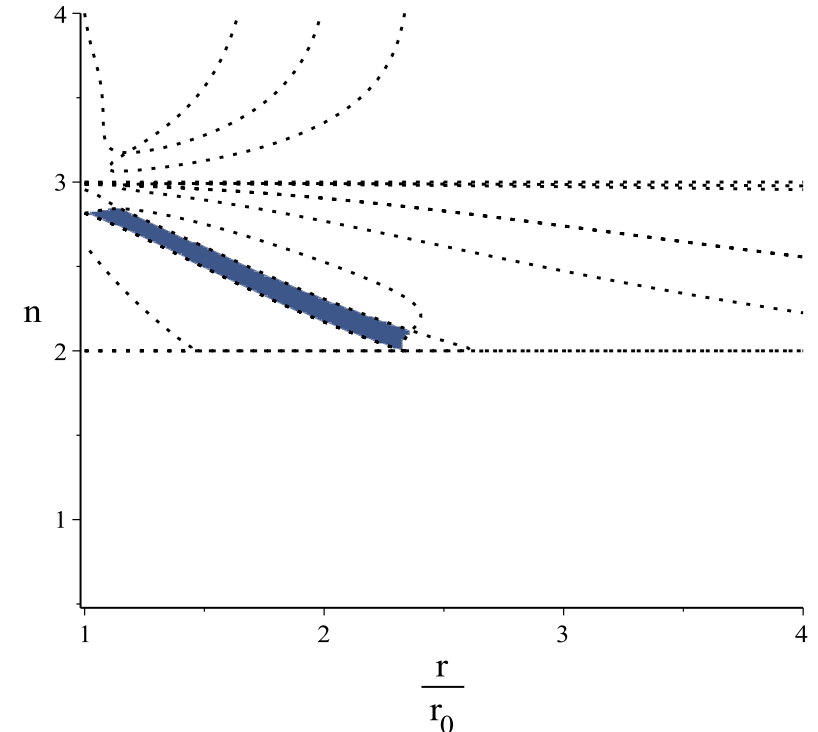

(b)

this model, respect NEC by choosing adequate values for $n$. In these figures we set $m=2, a=2, b=20, c=20$ and $r_{0}=1$

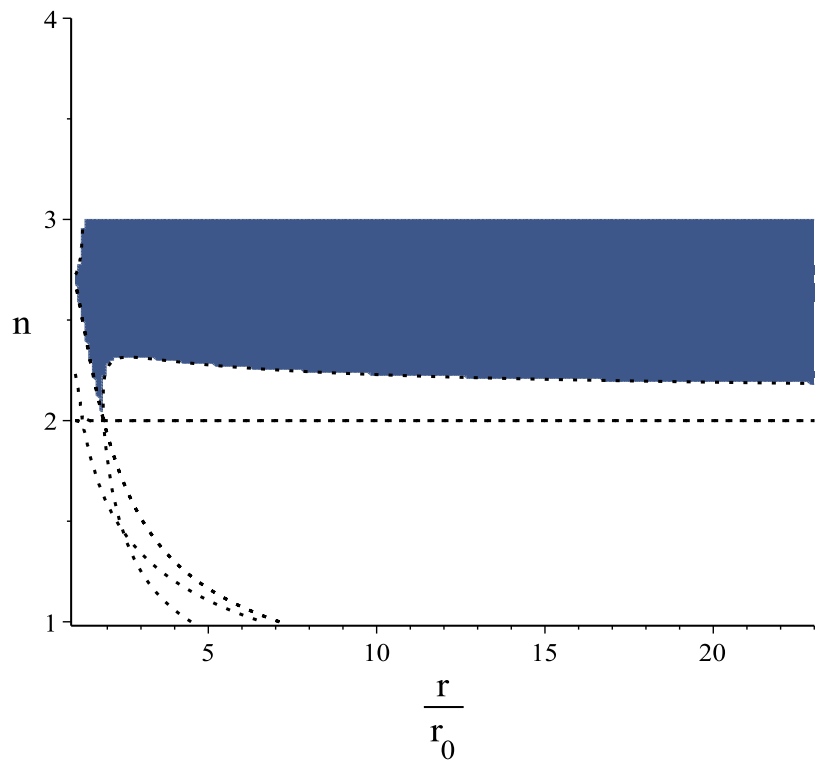

(b)

Fig. 6 The NEC for a wormhole solution in the case of Amendola-Gannouji-Polarski-Tsujikawa model is respected (a). In the blue part in b, traversable asymptoticly flat wormholes respect the NEC. In these figures we set $\mu=5, R_{*}=0.01, p=0.5$ and $r_{0}=3$

3) The Starobinsky model $[65,67,68]$

Another viable modified $f(R)$ gravity model that we consider, has three free parameters $\lambda, R_{*}$ and $q$ as:

$f(R)=R+\lambda R_{*}\left[\left(1+\frac{R^{2}}{R_{*}^{2}}\right)^{-q}-1\right]$ where $0.944<\lambda<0.966$ for $q=2$ according to [63] and $R_{*}$ takes small positive values. Similar to the previous case, we are looking for the wormhole solutions of this $f(R)$ model which respect the WEC (18). One can check that by choosing $0.944<\lambda<0.966, F$ in (20) takes positive values and there is no static wormhole solution in this model. However 


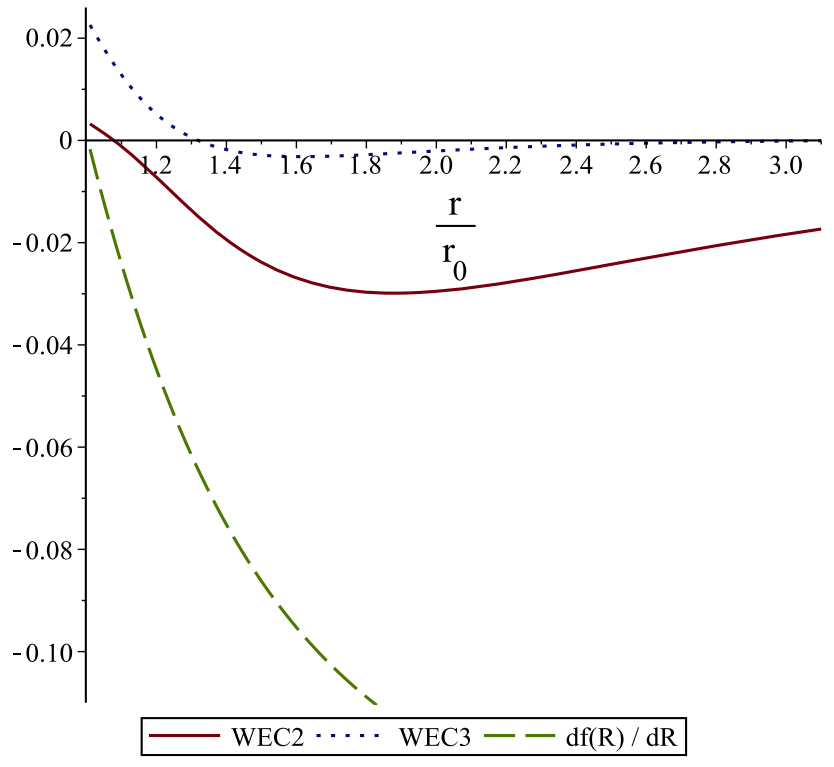

(a)

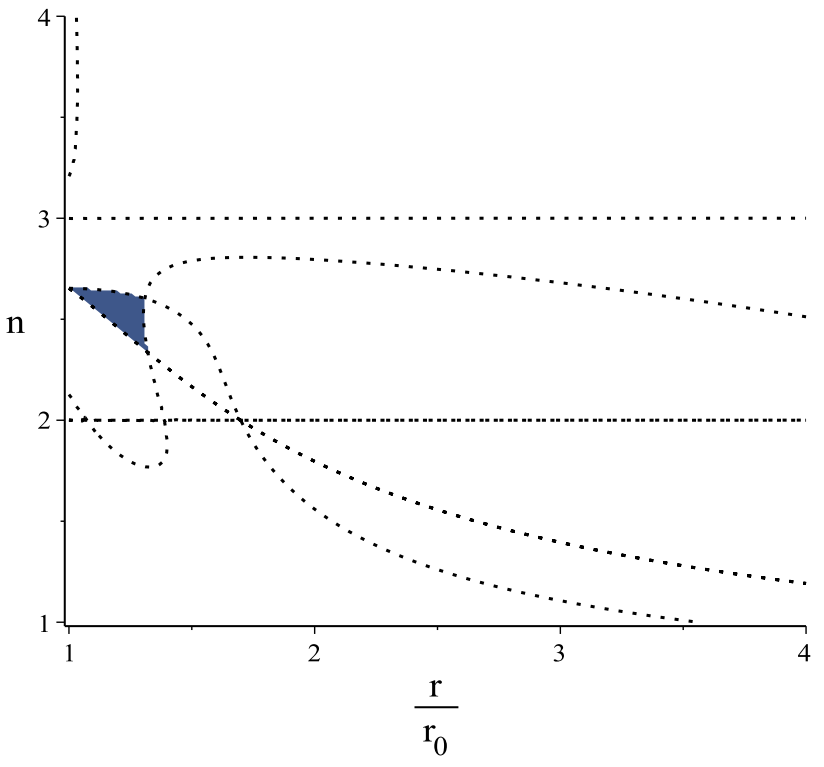

(b)

Fig. 7 Setting $n=2.65$ in a we found a wormhole solution in the exponential gravity model that respect NEC. The blue region in $\mathbf{b}$ also shows that it is possible to find wormholes solutions in this model that respect NEC around the throat. In these figures we set $\lambda=2, R_{*}=0.01$ and $r_{0}=10$

by taking $\lambda$ at the neighborhood of this range, it is possible to obtain negative values for $F$. In Fig. 4 a we draw $F$ and (18) by choosing $\lambda=0.98, n=2.5$. It is clear that by this choice, $F$ is negative at the throat however the null (and so weak) energy condition is violated at the wormhole throat.

We also tried - by varying the parameter $n$ - to find wormhole solutions that respect NEC, but there is no region in $n-r / r_{0}$ diagram that $F<0$ and the NEC is satisfied simultaneously (the $n-r / r_{0}$ diagram is blank). In the other words, our results show that there is no asymptotically flat wormhole in this model that respect the NEC.

4) Nojiri-Odintsov model [69]

Another viable $f(R)$ model suggests that

$f(R)=R+\frac{R^{m}\left(a R^{m}-b\right)}{1+c R^{m}}$,

where $m \geq 2$ and $a, b$ and $c$ are free positive parameters which satisfy the condition $b c>>a$. Setting $m=2, a=$ $2, b=20, c=20$ and $n=2.8$, we checked that $F<0$ and WEC2, WEC 3 are respected around the wormhole throat $r_{0}$ (Fig. 5a). Figure 5b shows that the $f(R)$ model (24) contains asymptoticly flat wormhole solutions with different values for the parameter $n$, that respect the null energy condition.

5) Amendola-Gannouji-Polarski-Tsujikawa model In the fifth model that we consider, the $f(R)$ is in the form $[65,70]$

$f(R)=R-\mu R_{*}\left(R / R_{*}\right)^{p}$.
This viable model contains three parameters $0<p<1$, $\mu>0$ and $R_{*}$ which takes a small positive value. The same analysis by setting $p=1 / 2, \mu=5$ and $n=2.75$, shows that $F$ in (20) becomes negative and the obtained wormhole solution satisfies NEC at the throat (Fig. 6a). It is also obvious in Fig. $6 \mathrm{~b}$ that there are asymptoticly flat traversable wormhole solutions in this model which respect NEC outside the throat $r_{0}$, if one choose adequate values for $n$.

6) Exponential gravity model $[71,72]$

For the last model that we consider in this section, the $f(R)$ is in the form

$f(R)=R-\lambda R_{*}\left[1-\exp \left(-\frac{R}{R_{*}}\right)\right]$,

where $\lambda, R_{*}$ are free positive parameters of the model. Similar to the previous cases, one can find a wormhole in this model which respect NEC at the throat (Fig. 7a). In Fig. 7b the $n-r / r_{0}$ diagram is depicted. It is clear that there is a little possibility for traversable asymptotically flat wormhole solutions in this model to satisfy the null energy conditions around the throat $r_{0}$.

\subsection{The cases of $c_{1} \neq 0$}

We considered traversable wormhole solution as (6), where $b(r) / r$ given by (17). Remember that the wormhole shape function $b(r)$ satisfies the conditions in (15) which leads to $n>2$ in the case of $c_{1}=-1$ and $n>2.4$ in the case of $c_{1}=1$, therefore the second term in (17) falls down at 


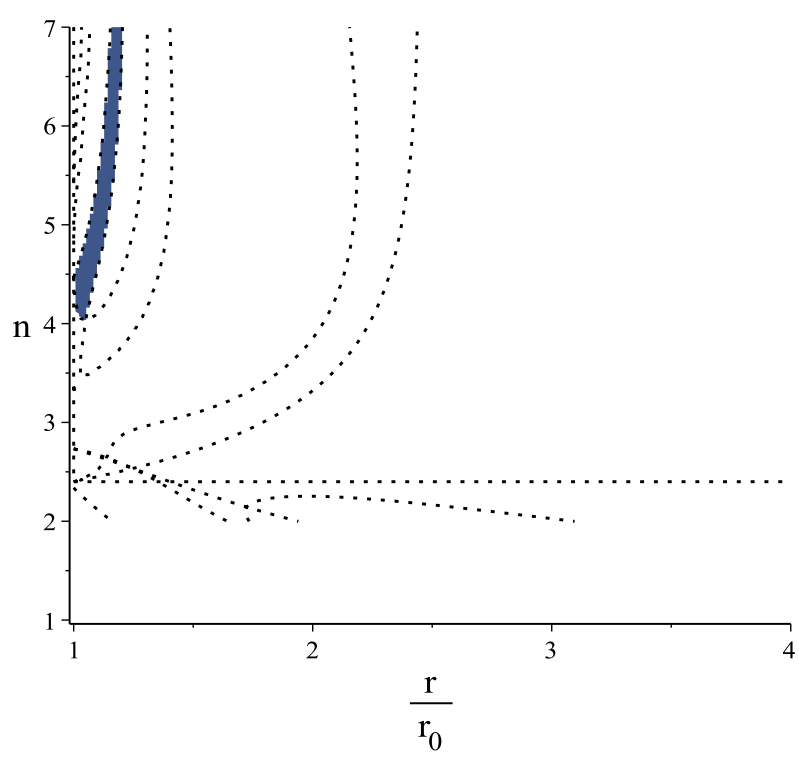

(a)

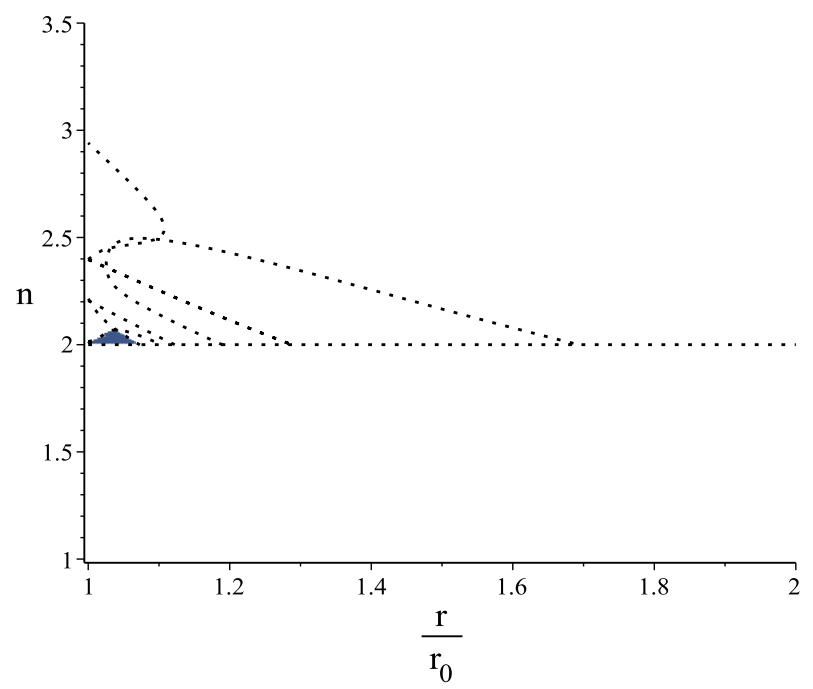

(c)

Fig. 8 The blue region in a, $\mathbf{c}$ show the asymptotically spherical (hyperbolic) wormhole solutions of the Tsujikawa model that respect the NEC. b Which is depicted by setting $n=4.5, r_{0}=1$, shows that $F=d f(R) / d R<0$ and the asymptotically spherical wormhole

large $r$. In this subsection we choose $c_{1}= \pm 1$, for which the wormhole metric (6) at large $r$ matches hyperbolic $\left(c_{1}=-1\right)$ and spherical $\left(c_{1}=1\right)$ FRW metric, so we call them asymptotically hyperbolic and asymptotically spherical wormhole solutions respectively. In the following we verify the null and weak energy condition for these asymptotically hyperbolic and spherical wormhole solutions in the background of static $f(R)$ modified gravity models that we considered in the previous subsection.

1) The Tsujikawa model

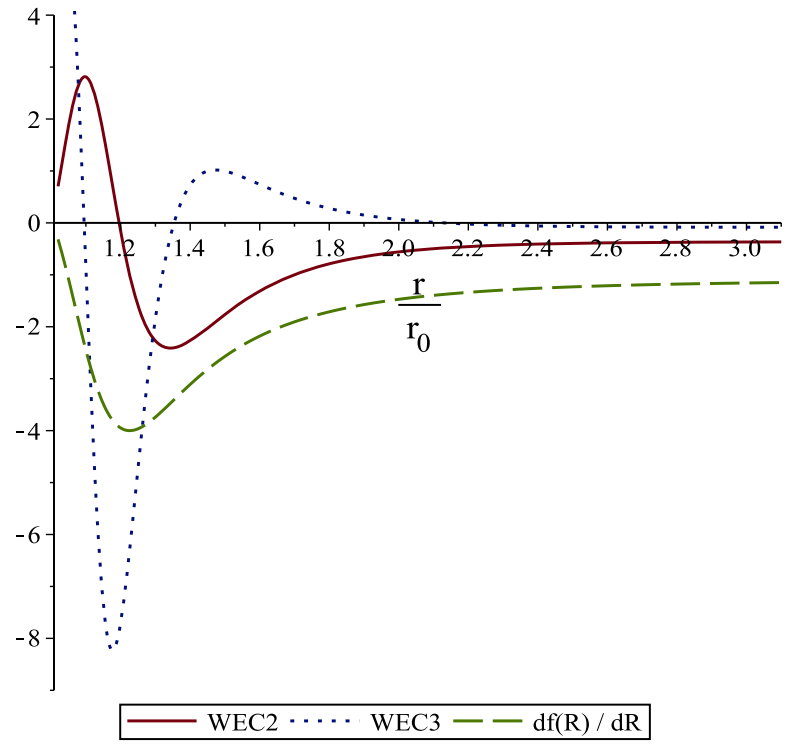

(b)

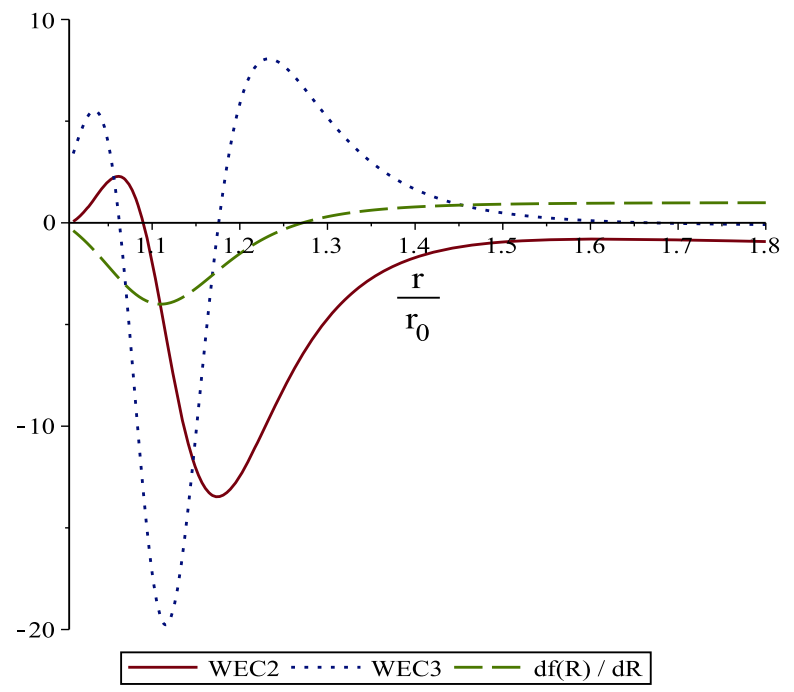

(d)

in this model respect NEC around the throat. $\mathbf{d}$ Demonstrates that for an asymptotically open wormhole with $n=2.02, r_{0}=0.5$, NEC is respected around the throat. In these figures we set $\mu=5, R_{*}=1$

For this model the $f(R)$ is given in (21). We are interested in solutions for which $F$ in (20) takes negative values, conditions $i i$ ), iii in (15) are satisfied and (18) are respected simultaneously. Figure 8a, c show the location of these asymptotically closed $\left(c_{1}=1\right)$ and open $\left(c_{1}=-1\right)$ wormhole solutions in the $n-r / r_{0}$ diagram respectively. In Fig. 8b, d we have explicitly depicted $F$ and requirements of the WEC for an asymptotically spherical (hyperbolic) wormhole solution with $n=4.5$ ( $n=2.02$ ). It is obvious that by choosing adequate values for the parameters, one can find traversable 


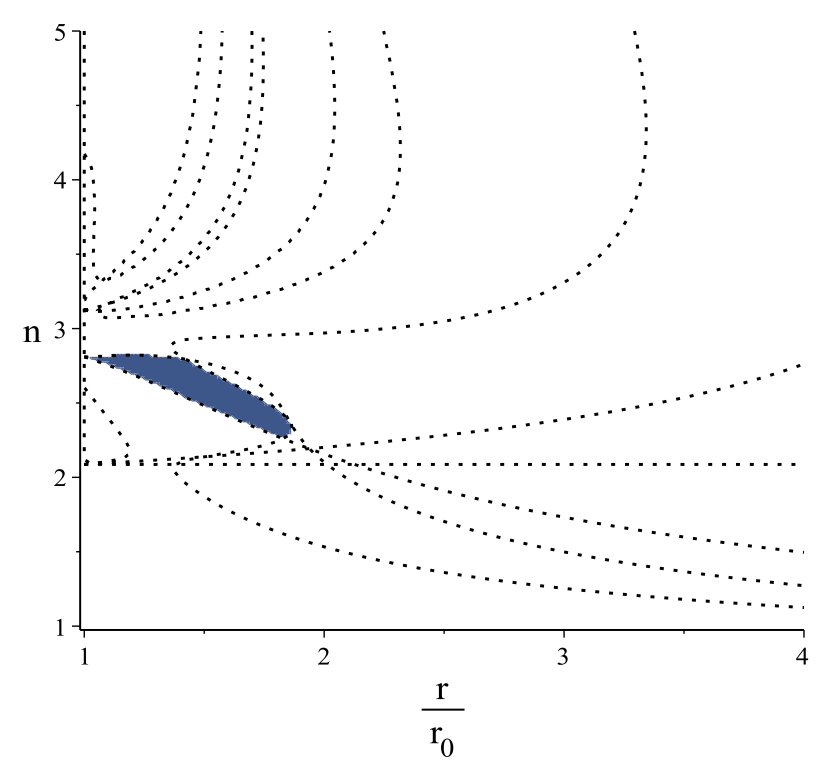

(a)

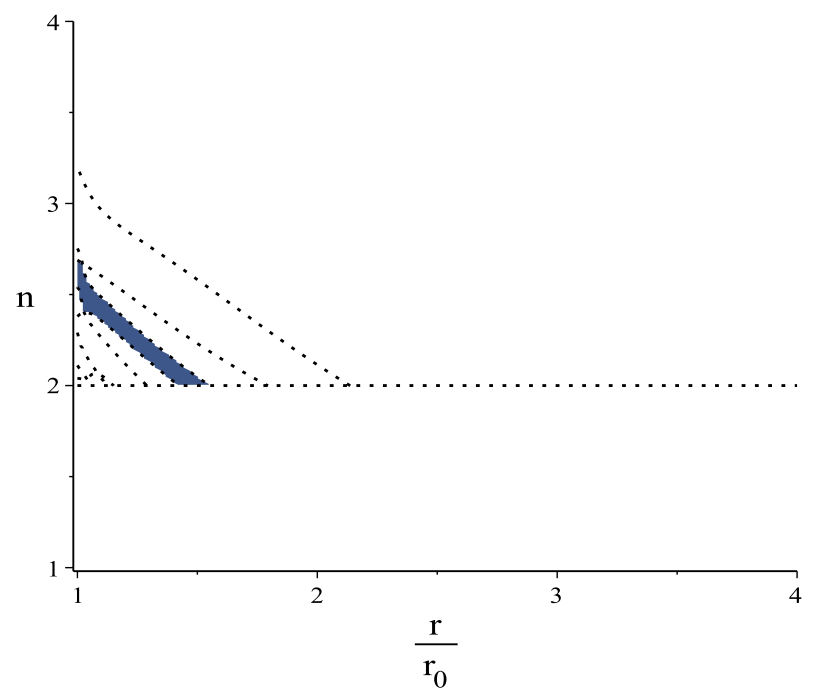

(c)

Fig. 9 Similar to the previous model, there are asymptotically spheri$\mathrm{cal}$ (a) and hyperbolic (c) wormholes in $\mathrm{Hu}$-Sawicki model that respect the NEC. In b we draw $F$ and WEC2,3 for an asymptoticly spherical

asymptotically closed and open wormhole solutions in this $f(R)$ gravity model that respect NEC around the wormhole throat.

\section{2) Hu-Sawicki model}

The second model that we studied its wormhole solutions at the previous subsection was the $\mathrm{Hu}-$ Sawicki model with the $f(R)$ in the form (22). We verify the NEC for asymptotically spherical and hyperbolic wormhole solutions of this model in Fig. 9a, c respectively. In the blue regions in these figures $F=d f(R) / d R$ becomes negative, $i i)$, iii) of (15) are satisfied and (18) are respected simultaneously. We have

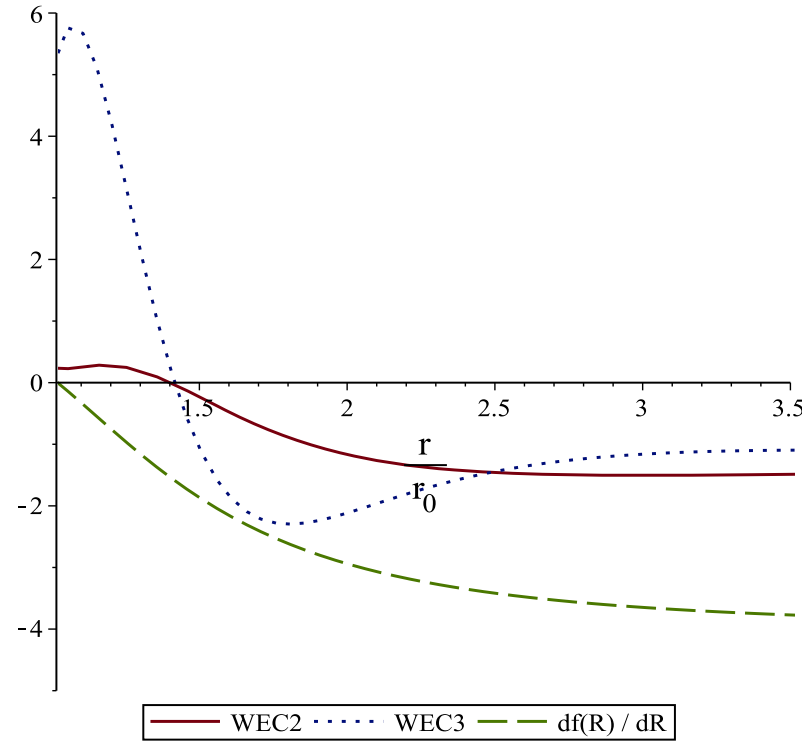

(b)

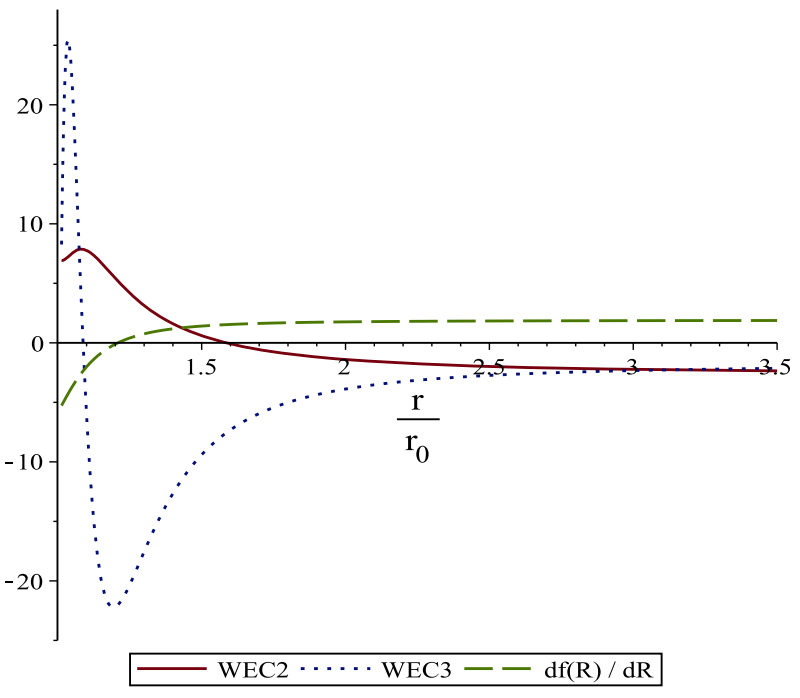

(d)

solution with $n=2.8$. d Also shows that an asymptoticly hyperbolic wormhole with $n=2.5$, respect the NEC. In these figures we set the parameters as $\mu=10, R_{*}=1, r_{0}=.5$

plotted the NEC for an asymptotically spherical and hyperbolic wormhole solution in Fig. 9b, d respectively.Similar to the previous model, it is clear that by setting the parameters in adequate values, traversable asymptotically spherical and hyperbolic wormholes in this model respect the null energy condition around the throat $r_{0}$.

3) The Starobinsky model

In the case of Starobinsky model which $f(R)$ is given in (23), the $d f(R) / d R<0$ could be satisfied for both asymptotically spherical and hyperbolic solutions by choosing $R_{*}$ and $r_{0}$ appropriately, however the null energy condi- 


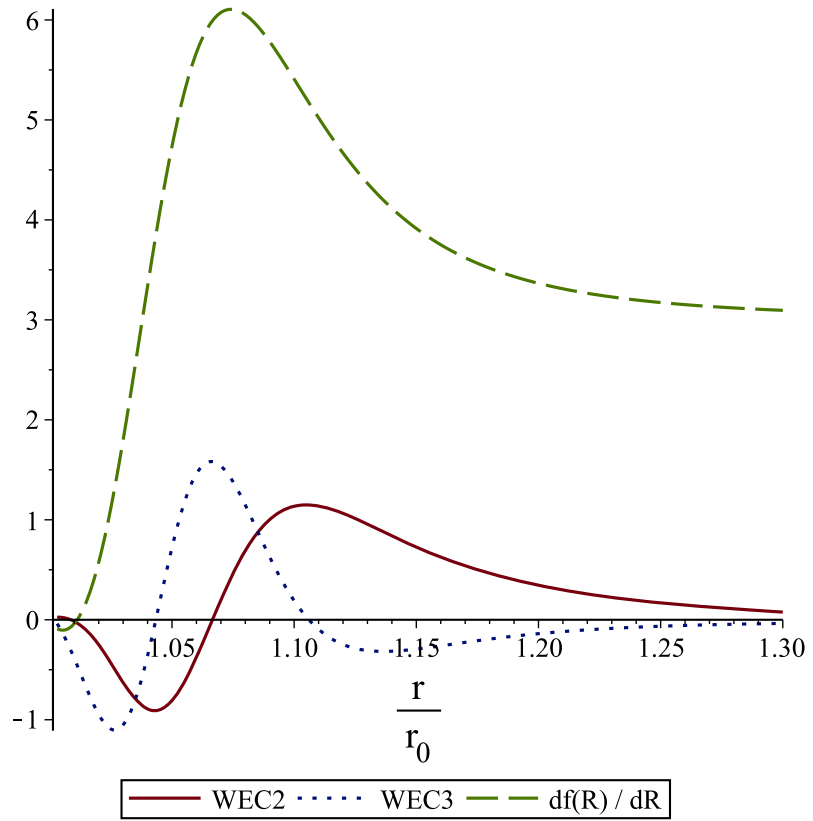

(a)

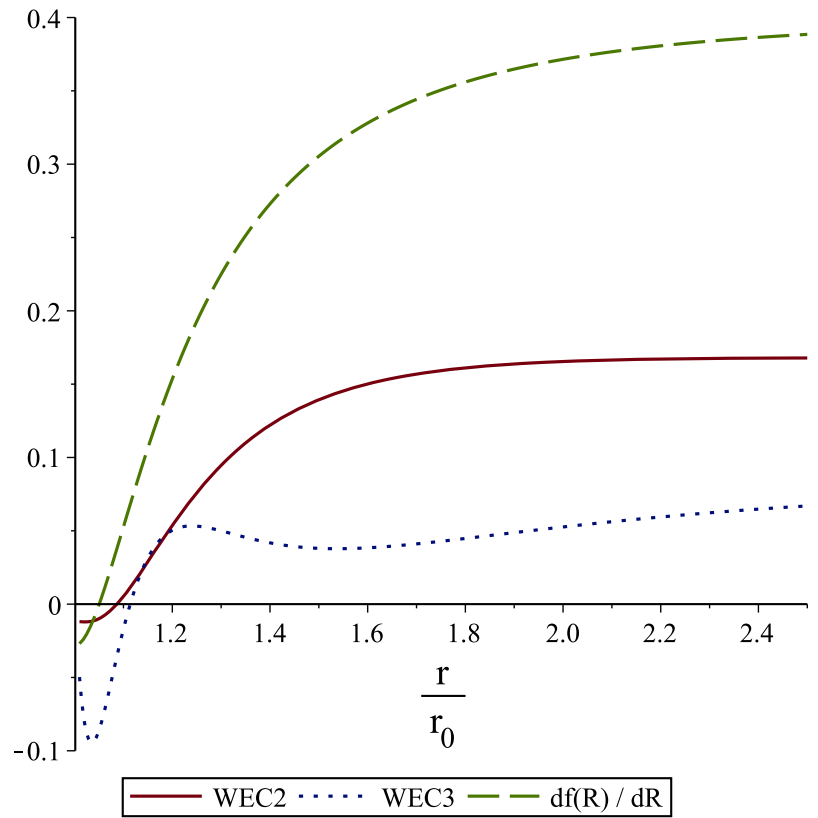

(b)

Fig. 10 In the case of Starobinsky model, a, b show $F$, WEC2,3 for the asymptotically hyperbolic and spherical wormhole solutions respectively. In both cases by fixing $\lambda=1, R_{*}=1$ the $F<0$ is satisfied around $r_{0}$, however the NEC are violated. We set $r_{0}=0.55, n=2.25$ in a and $r_{0}=1.5, n=4$ in $\mathbf{b}$

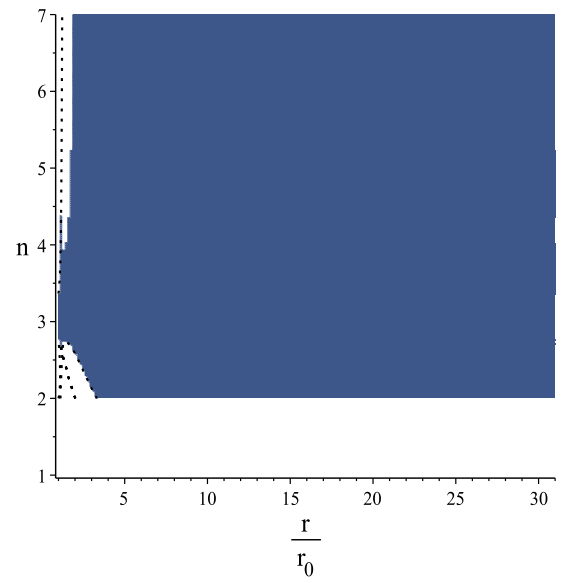

(a)

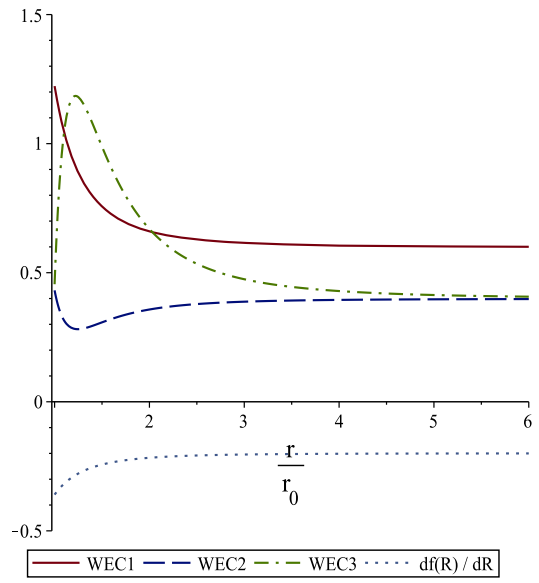

(b)

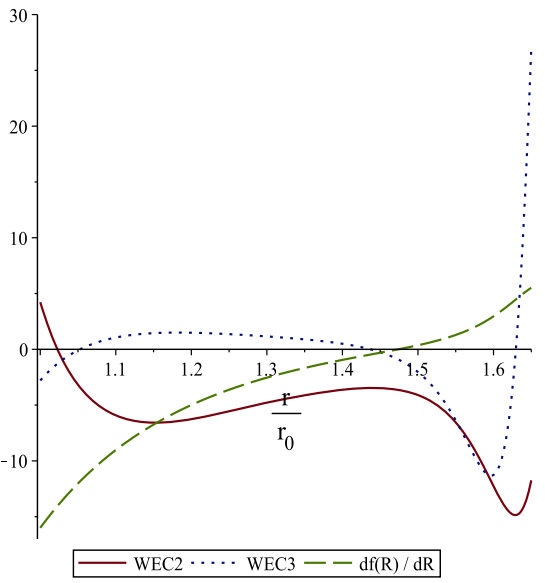

(c)
Fig. 11 a Shows that the asymptoticly hyperbolic wormholes with $n>2$ in the Nojiri-Odintsov model, respect the WEC almost through the whole space outside the throat $r_{0}$. We have depicted WEC1,2,3 and $F$ for an asymptotically hyperbolic wormhole with $n=3.2$ in $\mathbf{b}$. The asymptoticly spherical wormholes do not respect the NEC in this background (c). In $\mathbf{a}, \mathbf{b}$ we set $a=2, b=20, c=20$ and $r_{0}=1$. c Is drawn by setting $a=5, b=20, c=10$ and $r_{0}=0.5$

diagram for asymptotically hyperbolic $\left(c_{1}=-1\right)$ solutions in this model by setting $m=2$, is depicted in Fig. 11a. The blue zone in this diagram corresponds to the region that $F<0, i i)$, iii) of (15) and (18) are satisfied simultaneously. It is clear that the asymptoticly hyperbolic wormholes with $n>2$ in this model, respect the WEC almost through the whole space outside the throat $r_{0}$. In Fig. $11 \mathrm{~b}$ we have drawn 


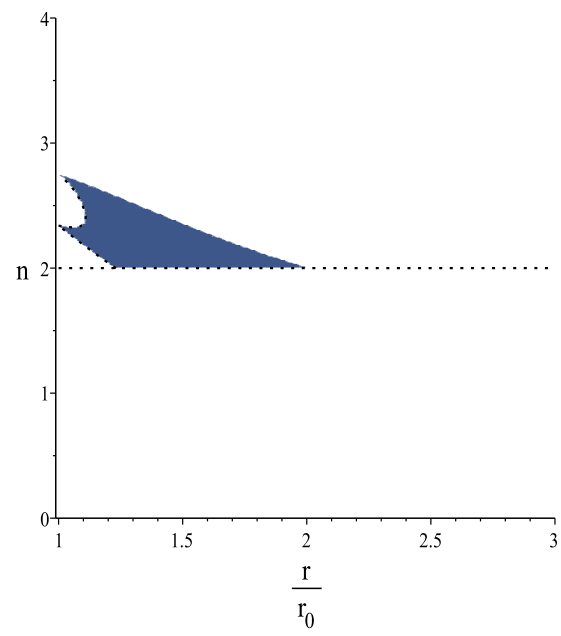

(a)

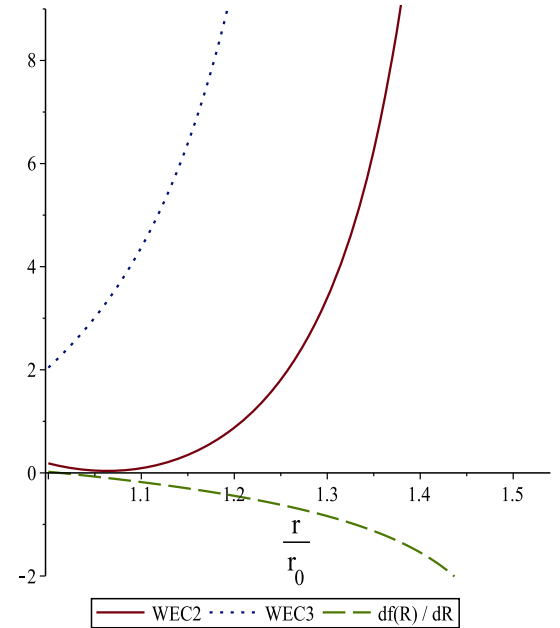

(b)

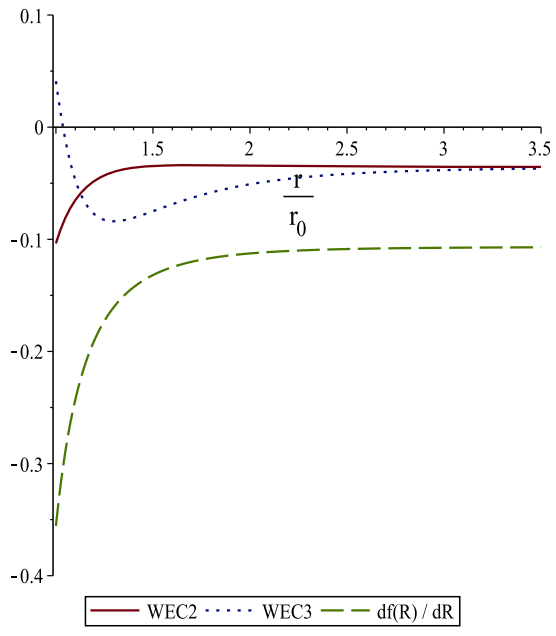

(c)
Fig. 12 The blue region in a corresponds to asymptoticly hyperbolic wormhole solutions that respect the NEC in Amendola-GannoujiPolarski-Tsujikawa model. It is clear from $\mathbf{b}$ that $F<0$ and NEC is respected around $r_{0}$, for an asymptoticly hyperbolic wormhole with $n=2.3$. In $\mathbf{c}$ by setting $n=5$ we show an example for violation of the NEC in the case of asymptotically spherical wormholes in this model. We set the parameters as $p=1 / 2, R_{*}=0.1, \mu=20, r_{0}=0.3(\mu=$ $\left.7, r_{0}=2\right)$ in $\mathbf{a}, \mathbf{b}(\mathbf{c})$

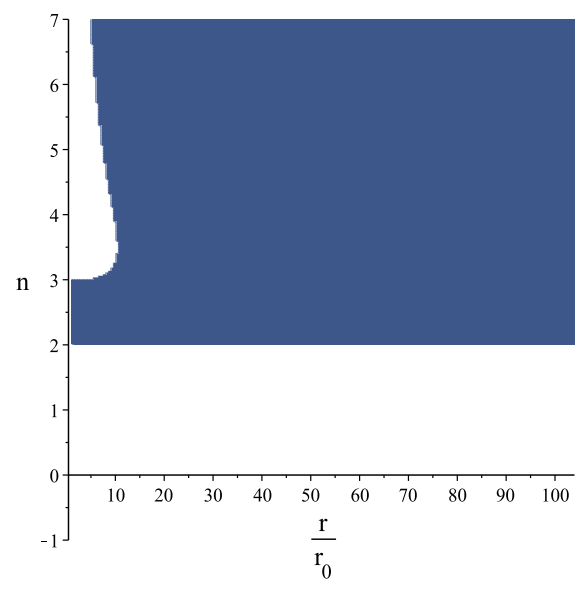

(a)

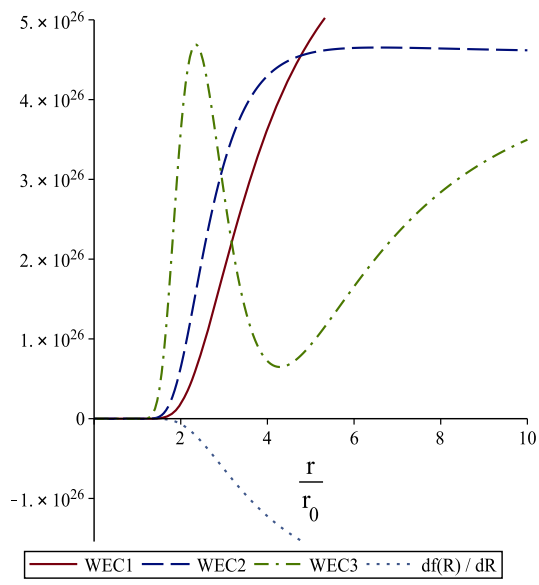

(b)

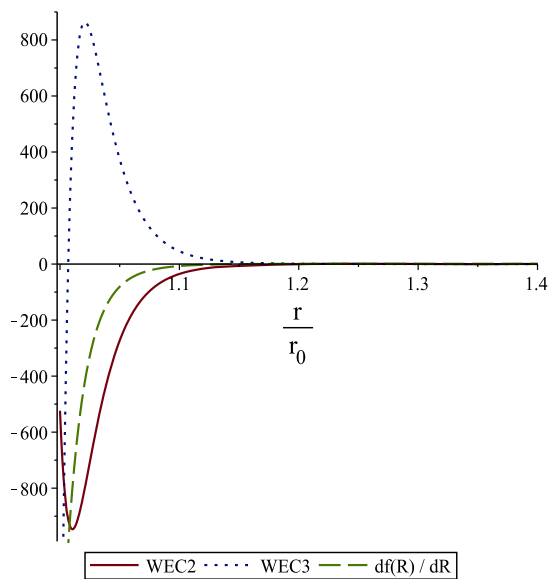

(c)
Fig. 13 In the case of $c_{1}=-1$, a shows the region that $F<0$ and (18) are satisfied in blue. This means that the WEC is respected by the asymptotically hyperbolic wormhole solutions of the exponential gravity model, almost through the whole space outside the throat. In $\mathbf{b}$ we

$d f(R) / d R$ and WEC1,2,3 explicitly for a wormhole solution with $n=3.2$.

In the case of $c_{1}=1$, it is possible to set the parameters in a manner that $F<0$ is satisfied, however these asymptotically spherical wormhole solutions do not respect the NEC (see Fig. $11 \mathrm{c}$ for instance), in the other words, the $n-r / r_{0}$ diagram in this case is blank.

5) Amendola-Gannouji-Polarski-Tsujikawa model

The $f(R)$ in this model is given by (25). In the case of $c_{1}=-1$ we check the NEC for wormhole solutions of have plotted $F$ and WEC1,2,3 for an asymptoticly hyperbolic wormhole with $n=2.5$. In the case of $c_{1}=1$, Fig (13.c) shows that the NEC is violated at the throat for a wormhole with $n=4$. In these figures we set $\lambda=2, R_{*}=0.1$ and $r_{0}=1$

this model in Fig. 12a which shows that it is possible to find asymptotically hyperbolic wormholes that respect NEC around the throat, by choosing appropriate parameters in this static model. In Fig. 12b we have explicitly drawn the NEC for a wormhole by setting $n=2.3$.

In the case of $c_{1}=1$ our results show that, like the previous model, there is no asymptoticly closed wormhole solution in this model which respect NEC (the $n-r / r_{0}$ diagram is blank). As an example we have depicted $F=d f(R) / d R$ and WEC2,3 for a wormhole by setting $n=5$ in Fig. 12c. 
6) Exponential gravity model

For the last model that we considered in the previous subsection, the $f(R)$ is given by (26). The same analysis shows that in the case of $c_{1}=-1$, there are asymptotically hyperbolic wormhole solutions which respect the WEC almost through the whole space outside the wormhole throat (Fig. 13a, b). It is also possible in the case of $c_{1}=1$, to find asymptoticly spherical wormhole solutions by choosing parameters in a manner that $F<0$. However the NEC are violated by these wormholes as we can see in the Fig. 13c.

\section{Conclusion}

In this paper we considered wormhole solutions in the context of some $f(R)$ modified gravity models. In the first step, by solving the field equations similar to [62], we found the energy density, radial and transverse pressure (10)-(12) of the solutions in terms of $F=d f(R) / d R$ and the shape function $b(r)$. In the second step we considered the Ricci scalar of the wormhole solutions in the form (13) and reorganized the shape function into the form $b(r)=\left[\left(-r_{0}^{n} c_{1}+r_{0}^{n-2}\right) r^{3-n}+c_{1} r^{3}\right]$.

As the next step we inserted the physical conditions on $b(r)$ and found that $n$ must be greater than 2 . This convey us to choose the values $c_{1}=-1,0,+1$ for which the wormhole solution (6) at large radial coordinate $r$, matches the hyperbolic, flat and spherical FRW universe respectively (asymptotically hyperbolic, flat and spherical solutions). In the final step we chose six static $f(R)$ modified gravity models and checked whether the null and weak energy conditions (18) hold for the wormhole solutions in the background of these $f(R)$ models.

Our results show that by choosing adequate parameters, it is possible to find traversable asymptotically hyperbolic and flat wormhole solutions that respect NEC around the throat $r_{0}$, in all these $f(R)$ models except that the Starobinsky model. In the case of the Tsujikawa model, asymptoticly flat wormholes can also respect the weak energy condition through the whole space outside the throat. In the case of exponential gravity and Nojiri-Odintsov models, asymptoticly hyperbolic wormholes can respect WEC in the whole space outside the $r_{0}$ too. We also found that asymptotically spherical wormhole solutions respect the NEC around $r_{0}$ just in the Tsujikawa and Hu-Sawicki models. It is worth to mention that due to $F=d f(R) / d R<0$ for these wormhole solutions, the "remote mouths" would be located in antigravitational regions because of the effective gravitational constant is negative $[60,61]$.

As a final remark we emphasis that unlike the Einstein gravity, our results show that it is possible to find traversable wormhole solutions which respect the null and weak energy conditions in the background of some $f(R)$ modified gravity models. This means that $f(R)$ gravity in addition to its applications in cosmology, can be a useful framework in wormhole physics.

Acknowledgements H.G. would like to thank M.M. Sheikh-Jabbari, Ghadir Jafari and Davood Mahdavian Yekta for useful discussions.

Data Availability Statement This manuscript has no associated data or the data will not be deposited. [Authors' comment: The required data is given in the text of the paper.]

Open Access This article is distributed under the terms of the Creative Commons Attribution 4.0 International License (http://creativecomm ons.org/licenses/by/4.0/), which permits unrestricted use, distribution, and reproduction in any medium, provided you give appropriate credit to the original author(s) and the source, provide a link to the Creative Commons license, and indicate if changes were made.

Funded by SCOAP ${ }^{3}$.

\section{References}

1. L. Flamm, Phys. Z. 17, 448 (1916)

2. A. Einstein, N. Rosen, Phys. Rev. 48, 73 (1935)

3. M.S. Morris, K.S. Thorne, Am. J. Phys. 56, 395 (1988)

4. M.S. Morris, K.S. Thorne, U. Yurtsever, Phys. Rev. Lett. 61, 1446 (1988)

5. S. Kar, D. Sahdev, Phys. Rev. D 53, 722 (1996)

6. A.V.B. Arellano, F.S.N. Lobo, Class. Quantum Gravity 23, 5811 (2006)

7. M. Cataldo, P. Meza, P. Minning, Phys. Rev. D 83, 044050 (2011)

8. M.R. Mehdizadeh, M.K. Zangeneh, F.S.N. Lobo, Phys. Rev. D 92(4), 044022 (2015). arXiv:1506.03427 [gr-qc]

9. S.H. Mazharimousavi, M. Halilsoy, Z. Amirabi, Phys. Rev. D 81, $104002(2010)$

10. S.H. Mazharimousavi, M. Halilsoy, Z. Amirabi, Classical Quantum Gravity 28, 025004 (2011)

11. M.R. Mehdizadeh, M.K. Zangeneh, F.S.N. Lobo, Phys. Rev. D 92, 044022 (2015)

12. A.G. Agnese, M. La Camera, Phys. Rev. D 51, 2011 (1995)

13. K.K. Nandi, A. Islam, J. Evans, Phys. Rev. D 55, 2497 (1997)

14. F.S.N. Lobo, M.A. Oliveira, Phys. Rev. D 81, 067501 (2010)

15. S.V. Sushkov, S.M. Kozyrev, Phys. Rev. D 84, 124026 (2011)

16. E.F. Eiroa, G.F. Aguirre, Eur. Phys. J. C 72, 2240 (2012)

17. M. Richarte, C. Simeone, Phys. Rev. D 80, 104033 (2009)

18. M.R. Mehdizadeh, M.K. Zangeneh, F.S.N. Lobo, Phys. Rev. D 91, 084004 (2015)

19. M.K. Zangeneh, F.S.N. Lobo, M.H. Dehghani, Phys. Rev. D 92, 124049 (2015)

20. V.D. Dzhunushaliev, D. Singleton, Phys. Rev. D 59, 064018 (1999)

21. J.P. de Leon, J. Cosmol. Astropart. Phys. 11, 013 (2009)

22. R. Shaikh, S. Kar, Phys. Rev. D 94, 024011 (2016)

23. K.A. Bronnikov, A.M. Galiakhmetov, Gravit. Cosmol. 21(4), 283 (2015)

24. K.A. Bronnikov, A.M. Galiakhmetov, Phys. Rev. D 94(12), 124006 (2016)

25. M.R. Mehdizadeh, A.H. Ziaie, Phys. Rev. D 95, 064049 (2017)

26. C. Deffayet, G.R. Dvali, G. Gabadadze, Phys. Rev. D 65, 044023 (2002)

27. S.M. Carroll, V. Duvvuri, M. Trodden, M.S. Turner, Phys. Rev. D 70, 043528 (2004)

28. S. Nojiri, S.D. Odintsov, Phys. Rev. D 68, 123512 (2003)

29. A.A. Starobinsky, Phys. Lett. B 91, 99 (1980) 
30. V. Faraoni, Phys. Rev. D 72, 124005 (2005)

31. A. de la Cruz-Dombriz, A. Dobado, Phys. Rev. D 74, 087501 (2006)

32. N.J. Poplawski, Phys. Rev. D 74, 084032 (2006)

33. T. Clifton, J.D. Barrow, Phys. Rev. D 72, 103005 (2005)

34. S. Nojiri, S.D. Odintsov, Phys. Rev. D 74, 086005 (2006)

35. G. Cognola, E. Elizalde, S. Nojiri, S.D. Odintsov, S. Zerbini, Phys. Rev. D 73, 084007 (2006)

36. T. Harko, F.S.N. Lobo, M.K. Mak, S.V. Sushkov, Phys. Rev. D 87, 067504 (2013)

37. F.S.N. Lobo, A.I.P. Conf, Proc. 1458, 447 (2011)

38. F.S.N. Lobo, M.A. Oliveira, Phys. Rev. D 80, 104012 (2009)

39. N. Furey, A. De Benedictis, Class. Quantum Gravity 22, 313 (2005)

40. A. De Benedictis, D. Horvat, Gen. Relativ. Gravit. 44, 2711 (2012)

41. F. Rahaman, A. Banerjee, M. Jamil, A.K. Yadav, H. Idris, Int. J. Theor. Phys. 53, 1910 (2014)

42. M. Jamil, F. Rahaman, R. Myrzakulov, P.K.F. Kuhfittig, N. Ahmed, U.F. Mondal, J. Korean Phys. Soc. 65, 917 (2014)

43. S. Bhattacharya, S. Chakraborty, Eur. Phys. J. C 77, 558 (2017)

44. S. Bahamonde, M. Jamil, P. Pavlovic, M. Sossich, Phys. Rev. D 94, 044041 (2016)

45. E.F. Eiroa, G.F. Aguirre, Eur. Phys. J. C 78, 54 (2018)

46. E.F. Eiroa, G.F. Aguirre, Eur. Phys. J. C 76, 132 (2016)

47. E.F. Eiroa, G.F. Aguirre, Phys. Rev. D 94, 044016 (2016)

48. E.F. Eiroa, G.F. Aguirre, J.M.M. Senovilla, Phys. Rev. D 95, 124021 (2017)

49. T. Azizi, Int. J. Theo. Phys. 52, 3486 (2013)

50. N.M. Garcia, F.S.N. Lobo, Phys. Rev. D 82, 104018 (2010)

51. P. Pavlovic, M. Sossich, Eur. Phys. J. C 75, 117 (2015)

52. O. Bertolami, J. Paramos, Class. Quantum Gravity 25, 245017 (2008)

53. O. Bertolami, J. Paramos, Phys. Rev. D 77, (2008)

54. T. Multamaki, I. Vilja, Phys. Rev. D 73, 024018 (2006)

55. G.J. Olmo, Phys. Rev. D 75, 023511 (2007)

56. J.A.R. Cembranos, Phys. Rev. D 73, 064029 (2006)

57. W. Hu, I. Sawicki, Phys. Rev. D 76, 064004 (2007)

58. L. Sebastiani, L. Vanzo, S. Zerbini, Phys. Rev. D 97, 044009 (2018)

59. M. Calz, M. Rinaldi, L. Sebastiani, Eur. Phys. J. C 78, 178 (2018)
60. K.A. Bronnikov, A.A. Starobinsky, JETP Lett. 85, 1 (2007). Pisma Zh. Eksp. Teor. Fiz. 85, 3 (2007)

61. K.A. Bronnikov, M.V. Skvortsova, A.A. Starobinsky, Gravit. Cosmol. 16, 216 (2010)

62. F.S.N. Lobo, M.A. Oliveira, Wormhole geometries in $f(R)$ modified theories of gravity. Phys. Rev. D 80, 104012 (2009). arXiv:0909.5539 [gr-qc]

63. S. Tsujikawa, Phys. Rev. D 77, 023507 (2008). arXiv:0709.1391 [astro-ph]

64. A. De Felice, $S$. Tsujikawa, $f(R)$ theories. Living Rev. Relativ. 13, 3 (2010). arXiv: 1002.4928 [gr-qc]

65. L. Amendola, S. Tsujikawa, Dark energy theory and observations (Cambridge University Press, Cambridge, 2010)

66. W. Hu, I. Sawicki, Models of $f(R)$ cosmic acceleration that evade solar-system tests. Phys. Rev. D 76, 064004 (2007). arXiv:0705.1158 [astro-ph]

67. A.A. Starobinsky, Disappearing cosmological constant in $f(R)$ gravity. JETP Lett. 86, 157 (2007). arXiv:0706.2041 [astro-ph]

68. L. Amendola, D. Polarski, S. Tsujikawa, Are f(R) dark energy models cosmologically viable? Phys. Rev. Lett. 98, 131302 (2007). astro-ph/0603703

69. S. Nojiri, S.D. Odintsov, Modified $\mathrm{f}(\mathrm{R})$ gravity unifying $\mathrm{R} * * \mathrm{~m}$ inflation with Lambda CDM epoch. Phys. Rev. D 77, 026007 (2008). arXiv:0710.1738 [hep-th]

70. L. Amendola, R. Gannouji, D. Polarski, S. Tsujikawa, Conditions for the cosmological viability of $f(R)$ dark energy models. Phys. Rev. D 75, 083504 (2007). gr-qc/0612180

71. G. Cognola, E. Elizalde, S. Nojiri, S.D. Odintsov, L. Sebastiani, S. Zerbini, A class of viable modified $f(R)$ gravities describing inflation and the onset of accelerated expansion. Phys. Rev. D 77, 046009 (2008). arXiv:0712.4017 [hep-th]

72. E. Elizalde, S. Nojiri, S.D. Odintsov, L. Sebastiani, S. Zerbini, Non-singular exponential gravity: a simple theory for early- and late-time accelerated expansion. Phys. Rev. D 83, 086006 (2011). arXiv:1012.2280 [hep-th] 